\title{
Local boron environment in B-doped nanocrystalline diamond films $\dagger$
}

\author{
Stuart Turner, ${ }^{* a}$ Ying-Gang Lu, ${ }^{a}$ Stoffel D. Janssens, ${ }^{b c}$ Fabiana Da Pieve, ${ }^{a}$ Dirk Lamoen, ${ }^{a}$ Jo Verbeeck, ${ }^{a}$ \\ Ken Haenen, ${ }^{b c}$ Patrick Wagner ${ }^{b c}$ and Gustaaf Van Tendeloo ${ }^{a}$
}

\author{
Received 18th June 2012, Accepted 6th August 2012 \\ DOI: $10.1039 / \mathrm{c} 2 \mathrm{nr} 31530 \mathrm{k}$
}

Thin films of heavily B-doped nanocrystalline diamond (B:NCD) have been investigated by a combination of high resolution annular dark field scanning transmission electron microscopy and spatially resolved electron energy-loss spectroscopy performed on a state-of-the-art aberration corrected instrument to determine the B concentration, distribution and the local B environment. Concentrations of $\sim 1$ to 3 at.\% of boron are found to be embedded within individual grains. Even though most NCD grains are surrounded by a thin amorphous shell, elemental mapping of the B and C signal shows no preferential embedding of $\mathrm{B}$ in these amorphous shells or in grain boundaries between the NCD grains, in contrast with earlier work on more macroscopic superconducting polycrystalline Bdoped diamond films. Detailed inspection of the fine structure of the boron K-edge and comparison with density functional theory calculated fine structure energy-loss near-edge structure signatures confirms that the B atoms present in the diamond grains are substitutional atoms embedded tetrahedrally into the diamond lattice.

\section{Introduction}

Nanocrystalline diamond is a hugely attractive material because of its extreme hardness, chemically inert surfaces, high Young's modulus and large band gap of $5.5 \mathrm{eV}$ which can be readily modified by addition of dopants. Boron doping of NCD leads to a wide range of electronic transport properties with a critical boron concentration for the metal-insulator transition between $2 \times 10^{20}$ and $3 \times 10^{20} \mathrm{~cm}^{-3} \cdot \cdot^{-4}$ For boron concentrations above $10^{21} \mathrm{~cm}^{-3}$, B:NCD is also known to be superconducting below liquid helium temperatures. ${ }^{5}$

Much debate surrounds the question of the position and coordination of the $\mathrm{B}$ dopants in this type of material. It was recently shown using a combination of electron energy-loss spectroscopy (EELS) and energy-filtered TEM (EFTEM) that superconducting films with large micron sized grains exhibit pockets of amorphous carbon material at the diamond grain interfaces and triple points that are strongly enriched in boron. ${ }^{6}$ However this effect has never been verified in NCD films. The reasons for this are obvious; the relatively low $\mathrm{B}$ concentration, the small diamond grain size and the problems of specimen preparation for NCD diamond hinder the acquisition of detailed experimental data.

${ }^{a}$ EMAT, University of Antwerp, Groenenborgerlaan 171, B-2020 Antwerp, Belgium.E-mail: stuart.turner@ua.ac.be

${ }^{b}$ Institute for Materials Research (IMO), Hasselt University, Wetenschapspark 1, B-3590 Diepenbeek, Belgium

'IMOMEC, IMEC vzw, Wetenschapspark 1, B-3590 Diepenbeek, Belgium $\dagger$ Electronic supplementary information (ESI) available. See DOI: $10.1039 / \mathrm{c} 2 \mathrm{nr} 31530 \mathrm{k}$
In this work, conducting films of B-doped nanocrystalline diamond in both plan-view and cross-section orientation will be investigated by a combination of high resolution annular dark field scanning transmission electron microscopy (HR-ADFSTEM) and spatially resolved electron energy-loss spectroscopy (STEM-EELS) performed on a state-of-the-art aberration corrected instrument. Using these tools, the $\mathrm{B}$ concentration, distribution and the local $\mathrm{B}$ environment in this type of thin nanocrystalline diamond films will be determined. The coordination of $\mathrm{B}$ in the diamond film will be identified through comparison of the experimental EELS fine structure to density functional theory (DFT) calculated fine structure signatures.

\section{Experimental section}

\section{Diamond film growth}

The growth of the B:NCD layers on a Si substrate is described in detail elsewhere. ${ }^{7}$ In short: diamond growth was carried out in an ASTeX 6500 series MPECVD reactor on a Si substrate that was treated with a colloidal suspension of 5-10 nm detonation diamond. The temperature was monitored by a Williamson Pro92 dual-wavelength pyrometer. Growth was performed in a $\mathrm{CH}_{4} / \mathrm{H}_{2}$ plasma with a methane concentration of $3 \%$. Doping was induced by trimethyl boron gas $\left(\mathrm{B}\left(\mathrm{CH}_{3}\right)_{3}\right)$ with a $5500 \mathrm{ppm} \mathrm{B/C}$ ratio. The substrate temperature was $700{ }^{\circ} \mathrm{C}$ induced by $3500 \mathrm{~W}$ of microwave power at a total pressure of $60 \mathrm{hPa}$ (45 Torr). The growth was stopped when the B:NCD layers reached a thickness of $\sim 240 \mathrm{~nm}$ and cooling down was done under hydrogen flow. The thickness was determined ex situ, at standard ambient conditions, from SEM cross-sectional measurements. 


\section{TEM sample preparation}

TEM samples were prepared both in plan-view and in crosssection geometry. The samples were cut using a diamond wire, stuck together head-on (for the cross-section samples) and hand polished down to micrometer thickness. To thin the samples down to electron transparency, the samples were polished by ionmilling in a Balzers ion mill starting with a $8 \mathrm{kV}$ beam untill film perforation, continuing with a $5 \mathrm{kV}$ beam for thinning and a $2 \mathrm{kV}$ beam for final polishing.

\section{Scanning transmission electron microscopy and electron energy- loss spectroscopy}

High resolution STEM and spatially resolved EELS experiments were carried out on a FEI Titan 80-300 "cubed" microscope fitted with an aberration-corrector for the imaging lens and the probe forming lens, a monochromator and a GIF Quantum energy filter for spectroscopy, operated at $300 \mathrm{kV}$. ADF imaging was performed using a convergence semi-angle $\alpha$ of $\sim 21 \mathrm{mrad}$ and an ADF inner collection semi-angle of $22 \mathrm{mrad}$. STEMEELS experiments (Fig. 2 and 3) were performed using a convergence semi-angle $\alpha$ of $\sim 21 \mathrm{mrad}$ and a collection semiangle $\beta$ of $\sim 100 \mathrm{mrad}$, using a beam current of approximately $80 \mathrm{pA}$. All spectra were acquired at an energy dispersion of $0.1 \mathrm{eV}$ per pixel and an energy resolution of approximately $1.2 \mathrm{eV}$ (non-excited monochromator). The monochromator was not excited as, due to the low B content in the samples, sensitivity was the primary experimental concern.

EELS data in Fig. 4 was acquired in diffraction mode on a Philips CM 30 microscope operated at $300 \mathrm{kV}$ equipped with a GIF 200 spectrometer, using a convergence semi-angle $\alpha$ of $\sim 0.4$ $\mathrm{mrad}$ and a collection semi-angle $\beta$ of $\sim 0.9 \mathrm{mrad}$, at an energy dispersion of $0.1 \mathrm{eV}$ per pixel and an energy resolution of approximately $1.2 \mathrm{eV}$.

Chemical maps for the $\mathrm{C}$ signals (amorphous carbon and diamond) were generated by fitting the carbon K-edge to known references for diamond and amorphous carbon in the EELS model $^{8,9}$ software package. The B maps were generated by plotting the intensity under the background-subtracted B edge in each pixel using a $45 \mathrm{eV}$ broad energy window. The EELS data were first treated using principle component analysis (PCA) to minimize the influence of random noise. ${ }^{10}$ When plotted, the core-loss spectra were background subtracted, aligned using the onset of the graphitic peak (at $284 \mathrm{eV}$ ) and normalized in intensity to their maxima. Quantification of the EELS data was performed using Hartree-Slater cross-sections in Gatan Digital Micrograph.

\section{ELNES calculations using density functional theory}

$A b$ initio DFT-GGA (Generalized Gradient Approximation) calculations using the PBE (Perdew-Burke-Ernzerhof) functional were made using the all electron code WIEN2k, based on the full potential linearized augmented plane wave method. ${ }^{11,12}$ The following values for the relevant parameters were used: radius of the muffin tin $\left(R_{\mathrm{MT}}\right)=1.35, R_{\mathrm{MT}} \times \boldsymbol{K}_{\max }$ (basis cutoff, i.e., the product of the smallest muffin tin radius in the systems and the length of the maximum $\boldsymbol{K}$-vector of the interstitial plane wave basis) $=6.5 ; G_{\max }=14.0$.
The calculation of the doped system was performed on a $2 \times$ $2 \times 2$ supercell with 64 atoms, constructed from a pure diamond unit cell with lattice parameter $3.567 \AA$, allowing for a relaxation of the atomic positions and an isotropic expansion of the supercell. The final relaxed lattice parameter was $3.598 \AA$, slightly bigger than the experimental value, as expected from the GGA functional. The lattice parameters for the doped supercell were not relaxed, due to the reported small lattice expansion of boron doped diamond, especially in the case of substitutional impurities. ${ }^{13,14}$ The results for the relaxed atomic positions were in agreement with previous reports: the $\mathrm{B}$ impurity remained at the lattice site, and its neighbors relaxed away by $\sim 0.038 \AA$ with respect to pristine diamond, suggesting that the defect underwent a small Jahn-Teller distortion. ${ }^{15,16}$ The symmetry of the point defect remained $T_{\mathrm{d}}$, apart from a very small stretch $(0.001 \AA)$ of the $\mathrm{C}-\mathrm{B}$ bond along the $\langle 111\rangle$ direction with respect to the other $\mathrm{C}-\mathrm{B}$ bonds. The internal positions were also relaxed starting from an initially displaced configuration of the impurity, with the $\mathrm{B}$ atom displaced from the ideal position of the precursor $\mathrm{C}$ atom by $\sim 15 \%$ along the $\langle 111\rangle$ direction. This was done in order to rule out different stable final configurations for the small Jahn-Teller distortion reported for this defect, in a similar manner to calculations performed on other dopants in diamond in the literature. ${ }^{17}$ The differences in the total energy after optimization were of the order of $10^{-5} \mathrm{eV}$ and the final bond lengths did not differ, confirming the robustness of the relaxation.

The converged $\mathrm{k}$ mesh for the full Brillouin zone consisted of $2000 \mathrm{k}$ points for ground state calculations and $750 \mathrm{k}$ points for the $2 \times 2 \times 2$ supercell with 64 atoms used to calculate the ELNES. B K-edge ELNES calculations were performed using the full core hole approximation, i.e. introducing a frozen hole in the excited orbital and smearing the charge uniformly in space. No orientation dependence of the EELS spectra was considered, i.e. the cross-sections were averaged over all possible directions of the scattering vector with respect to the crystal, as no orientation relation of the $\mathrm{B}$ K-edge was observed in earlier XAS experiments. ${ }^{18}$ The structure was optimized allowing for a change of atomic coordinates with a convergence of the total energy of $2 \mathrm{meV}$. The calculated ELNES spectra were broadened to mimic instrumental broadening.

\section{Results and discussion}

Fig. 1 shows three ADF-STEM images of the B:NCD sample. ADF-STEM imaging was performed using an ADF collection inner semi-angle optimized to allow both diffraction and massthickness contrast to contribute to the image formation in order to make defects easily visible. ${ }^{19}$ The close packing of the diamond grains can be seen in the plan-view image in Fig. 1a. The diamond grains are well-distinguishable, and have sizes ranging from 100 to $300 \mathrm{~nm}$. Planar defects are abundant; many twin boundaries, typical of nanosized diamond, are visible in a single diamond grain in Fig. $1 \mathrm{~b}$ as well as in the diamond grains viewed in cross-section in Fig. 1c (indicated by arrows). The $71^{\circ}$ angle between the twin boundaries evidences that the twins are $\Sigma=3\{111\}$ planar defects. ${ }^{20,21}$ Individual diamond grains are generally surrounded by a thin amorphous shell. This is clearly visible in the plan-view sample; the diamond particle in Fig. 1b is surrounded by a lowcontrast, continuous amorphous carbon shell of $\sim 10 \mathrm{~nm}$ thickness. 

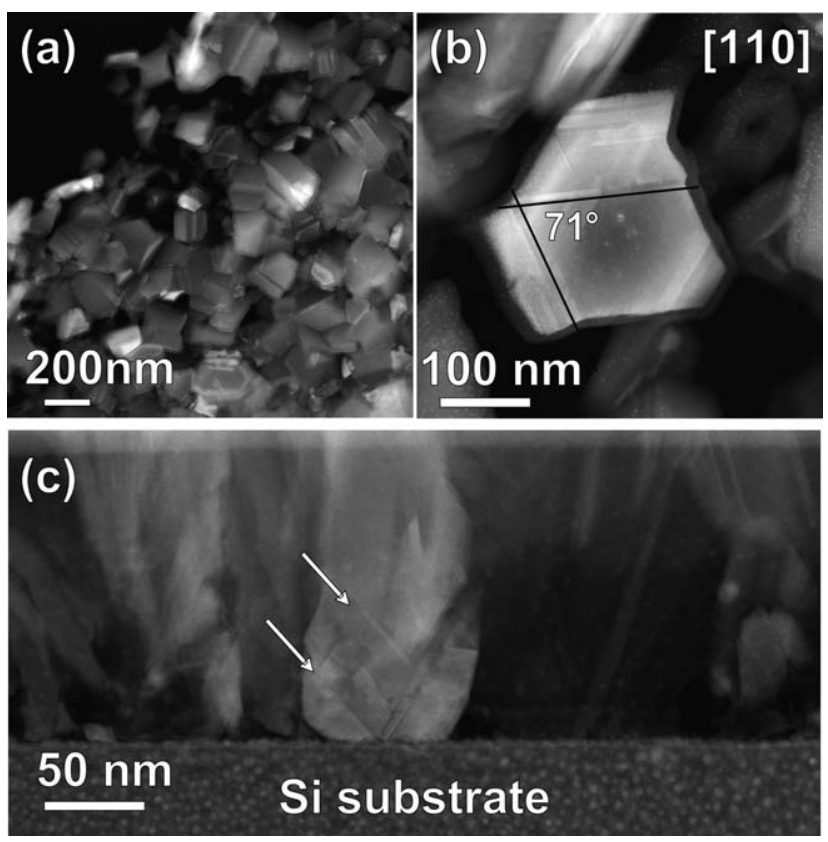

Fig. 1 ADF-STEM imaging of a B:NCD film; low magnification ADFSTEM image (a) showing diamond grains in the B:NCD film in planview. Higher magnification ADF-STEM images showing diamond grains with multiple $\{111\}$-type twin boundaries visible in plan-view (b) and cross-section (c, indicated by arrows). In some cases the diamond grains are surrounded by a thin amorphous shell (b).

No large triangular-shaped pockets are observed at the grain junctions, in contrast with previous observations in micron-sized polycrystalline boron-doped diamonds. ${ }^{6}$ In ultrananocrystalline diamond (UNCD) thin films, the morphology and electronic structure of grain boundaries (GBs) can be changed by the introduction of nitrogen. ${ }^{22}$ In work by Bhattacharyya et al. ${ }^{23} \mathrm{a}$ model is proposed in which carbon $\pi$ states in nitrogen-rich GBs are responsible for the high electrical conductivity.

In order to gain insight into how the boron dopants are distributed within the diamond grains, STEM-EELS measurements were performed on both cross-section and plan-view samples. To acquire the spectroscopic data, the so-called spectrum imaging technique was adopted. ${ }^{24}$ In this technique, the electron probe is scanned over the sample and an EELS spectrum is acquired in each point, together with an annular dark-field signal as image reference.

In Fig. 2, a tight aggregation of several NCD grains in crosssection is investigated by STEM-EELS. From the acquired EELS spectra, boron (Fig. 2b), diamond (Fig. 2c) and amorphous carbon (Fig. 2d) maps are generated. The ADF-STEM overview image in Fig. 2a does not show clear evidence for the presence of an amorphous carbon shell surrounding the individual diamond grains. However, the presence of such a shell is confirmed by the amorphous carbon map in Fig. 2d, with a thickness in the same order of magnitude as seen in Fig. 1b $(\sim 10 \mathrm{~nm})$. Even though the amorphous carbon map demonstrates the presence of amorphous carbon at the grain boundaries, no enrichment of boron at these grain boundaries is visible. On the contrary; the boron signal is evenly distributed over the diamond grains and grain boundary regions.
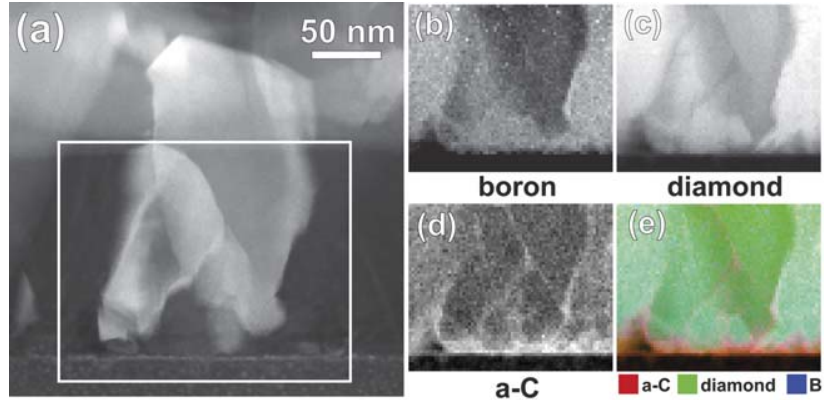

Fig. 2 Elemental mapping of the B:NCD film in cross-section; (a) ADFSTEM image of several diamond grains in cross-section orientation. (be) Elemental maps showing the boron (b), diamond (c) and amorphous carbon (d) distribution. (e) Color map.

Similar conclusions can be drawn from STEM-EELS data acquired from several diamond grains in plan-view orientation (Fig. 3). Again, the diamond grains are surrounded by an amorphous shell. The boron map shows no enrichment of B in the amorphous shell or at the grain boundaries between the NCD grains. A third set of STEM-EELS results obtained from a single NCD grain containing several planar defects (twins) is presented in the ESI. $\dagger$ Also here, no preferential enrichment of B in the amorphous carbon shell is detected. Even through enrichment of $\mathrm{B}$ at defect centers can be expected in these types of samples, no clear evidence for B enrichment at the twin boundaries can be derived from the data. Enrichment of B at defects in single diamond grains however remains a key question in the field and therefore warrants further investigation in the future. In conclusion, the STEM-EELS maps evidence the amorphous nature of the shell surrounding the individual diamond grains, but no specific boron enrichment at these boundaries is observed. The elemental maps showing the distribution of boron throughout the B:NCD films raise questions about the local environment of the B dopants.

To gain insight into this local environment/coordination of the B dopants, we performed electron energy-loss spectroscopy on
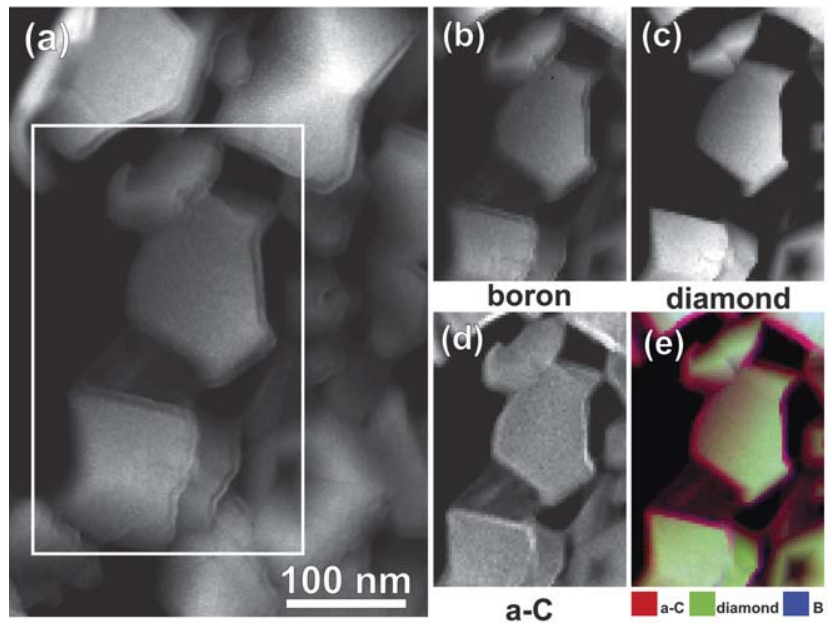

Fig. 3 Elemental mapping of the B:NCD film in plan-view; (a) ADFSTEM image of diamond grains in plan-view orientation. (b-e) Elemental maps showing the boron (b), diamond (c) and amorphous carbon (d) distribution. (e) Color map. 
both diamond and amorphous-rich regions. The fine structure of EELS edges (the so-called energy-loss near-edge structure (ELNES) signature) is known to be sensitive to the local environment of the excited atom through the projected density of unoccupied states. ${ }^{2,25}$ The ELNES signature of the boron $\mathrm{K}$-edge and carbon K-edge in these films should therefore contain information on the local B environment. To obtain a sufficient signal to noise ratio for ELNES investigation, EELS spectra were acquired from a diamond-rich and an amorphous carbon-rich region in diffraction mode, using longer acquisition times than for the STEM-EELS data.

In Fig. 4a, a typical EELS spectrum with the B K-edge at $190 \mathrm{eV}$ and the $\mathrm{C} \mathrm{K}$-edge at $284 \mathrm{eV}$ from a diamond-rich region is displayed. The $\mathrm{B}: \mathrm{C}$ ratio as measured from the spectrum is 2.2 at.\%. The B and C K-edge ELNES signatures for the diamondrich region are displayed in detail in Fig. 4b. The boron edge has been shifted over $94 \mathrm{eV}$ for comparison with the carbon edge. The overall ELNES signature for the carbon K-edge is typical for diamond, with a maximum peak coinciding with a $1 \mathrm{~s} \rightarrow \sigma^{*}$ transition at $292 \mathrm{eV}$, arising from the $\mathrm{sp}^{3}$ hybridization of $\mathrm{C}$ in diamond. The pre-peak at $285 \mathrm{eV}$ coincides with the $1 \mathrm{~s} \rightarrow \pi^{*}$ transition and is typical of $\mathrm{sp}^{2}$ hybridized carbon, which in this case is a small amount of amorphous carbon at the diamond grain boundaries.

Interestingly, the boron ELNES signature is highly similar to the carbon/diamond fine structure. Apart from a similar small $\pi^{*}$
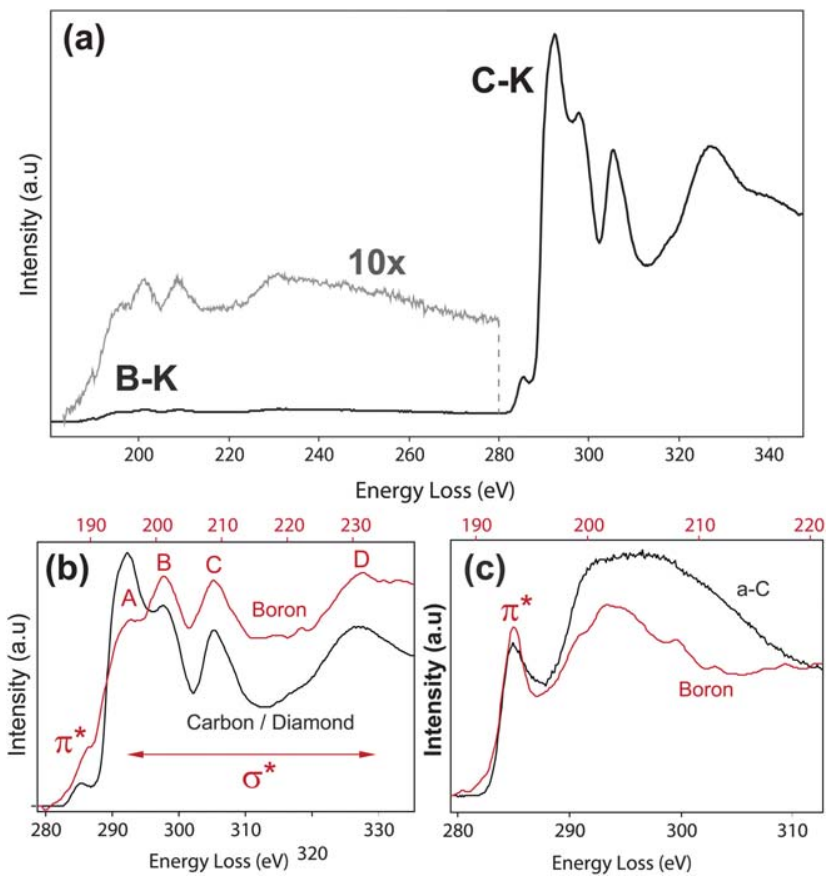

Fig. 4 EELS fine structure analysis of boron and carbon; (a) Typical EELS spectrum from a diamond grain region showing the boron K-edge at $190 \mathrm{eV}$ and the carbon K-edge at $284 \mathrm{eV}$. Quantification yields a B : C ratio of $2.2 \pm 0.2$ at.\%. (b) Comparison between a $2 \mathrm{eV}$ smoothed boron ELNES with the simultaneously acquired carbon ELNES from a diamond region and (c) comparison between the smoothed boron ELNES with the simultaneously acquired carbon ELNES from a amorphous carbon-rich region, scaled to match the carbon K-edge. The boron edges in (b) and (c) have been shifted to align with the carbon fine structure. Quantification yields a B : C ratio of $2.8 \pm 0.3$ at. $\%$. pre-peak, all four peaks A, B, C and D which are present in the diamond ELNES are also present in the boron fine structure. This implies that excited boron atoms are embedded in a similar local environment to the excited carbon atoms. In other words; the main fraction of boron atoms in the diamond-rich areas are present as substitutional impurities, which is consistent with previous XAS and XES spectroscopy results. ${ }^{18} \mathrm{~A}$ smaller fraction is embedded into the amorphous grain boundaries and shells. This is confirmed by the EELS data acquired from such an amorphous region (Fig. 4c). The boron K-edge from the amorphous carbon-rich region is characterized by a narrow $\pi^{*}$ peak followed by a broad $\sigma^{*}$ peak, similar to the fine structure of amorphous carbon. Quantification of the B : C ratio in this region yields a value of $2.8 \pm 0.3$ at. $\% \mathrm{~B}$, which is very close to the value in the diamond-rich region.

In an attempt to provide more accurate information on the type of boron embedding in the diamond grains, we performed detailed DFT calculations for the boron K-edge ELNES in the case of substitutional boron in diamond. One ELNES calculation was performed with full relaxation of the atomic positions surrounding the boron impurity; another was performed without relaxation (see Experimental section for full details). The results of the DFT calculations are plotted in Fig. 5 (top panel) together with the experimental boron K-edge and carbon K-edge fine structure from a diamond-rich region (bottom panel, replicated from Fig. 4b). After relaxation, the boron ELNES signature is found to be highly similar to the diamond ELNES, with peaks A, $\mathrm{B}$ and $\mathrm{C}$ being reproduced. Interestingly, a small pre-peak is

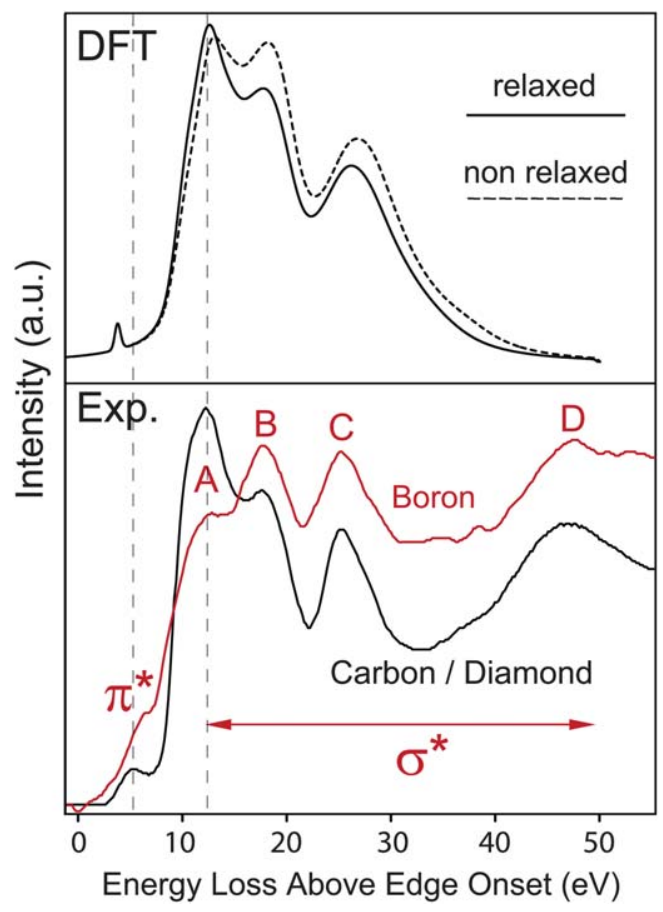

Fig. 5 Comparison between the DFT calculated ELNES and experimental data; top: DFT calculated B K-edge ELNES for substitutional B in diamond with structure relaxation (full line) and without internal structure relaxation (B at ideal $\mathrm{C}$ position; dotted line). Bottom: comparison between the $2 \mathrm{eV}$ smoothed boron ELNES with the simultaneously acquired carbon ELNES from a diamond region. The carbon edge has been shifted to align with the boron fine structure. 
present in the calculated boron spectrum, due to new states in the diamond band gap. This peak, $8.7 \mathrm{eV}$ before peak $\mathrm{A}$, is clearly present at a different energy-loss position than the $\pi^{*}$ peak, but has to date not been unequivocally observed experimentally. The ratio of the peaks A, B and C in the calculated ELNES signature does however not fully coincide with the experimental B ELNES, even though the peak ratios can in general be reproduced well for e.g. the carbon K-edge in diamond by DFT. ${ }^{26}$ The ratio changes of peaks $\mathrm{A}, \mathrm{B}$ and $\mathrm{C}$ are probably due to small changes in the local bond length, similar to the peak ratio changes seen in substitutional nitrogen in diamond. ${ }^{25}$ This is corroborated by the ELNES signature for the non-relaxed substitutional boron system; in this case the ratio of peaks A and B changes. This could indicate that the environment surrounding the $B$ impurities is not fully relaxed but rather strained or stressed, causing the peak ratios of peaks $\mathrm{A}, \mathrm{B}$ and $\mathrm{C}$ to change.

\section{Conclusions}

Thin films of B-doped nanocrystalline diamond have been investigated by a combination of high resolution annular dark field scanning transmission electron microscopy and spatially resolved electron energy-loss spectroscopy to determine the B concentration, distribution and the local B environment.

Concentrations of $\sim 1$ to 3 at. $\%$ of boron were found to be embedded tetrahedrally into the core of the NCD grains. Elemental mapping of the $\mathrm{B}$ and $\mathrm{C}$ signals showed no preferential embedding of $\mathrm{B}$ in grain boundaries or amorphous shells surrounding the diamond grains, in contrast to earlier work in literature. ${ }^{27,28}$ Detailed inspection of the fine structure of the boron K-edge and comparison with DFT calculated fine structure ELNES signatures confirms that the B atoms present in the diamond grains are substitutional atoms embedded tetrahedrally into the diamond lattice.

\section{Acknowledgements}

S.T., K.H. and J.V. gratefully acknowledge financial support from the Fund for Scientific Research Flanders (FWO) for a postdoctoral grant and under grant number G056810N. D.L, J.V and F.D.P gratefully acknowledge financial support from the GOA project "XANES meets ELNES" of the research fund of the University of Antwerp, Belgium. GVT acknowledges the ERC grant COUNTATOMS. The microscope used in this study was partially financed by the Hercules Foundation. The authors acknowledge support from the European Union under the Framework 7 program under a contract from an Integrated Infrastructure Initiative (Reference 262348 ESMI). Computing time was provided by the University of Antwerp (calcUA), Vlaams Supercomputercentrum (VSC) and the Hercules foundation. This work was financially supported by the EU FP7 Marie Curie ITN "MATCON" (PITN-GA-2009-238201), and the Collaborative Project "DINAMO" (no. 245122). GVT and PW acknowledge the methusalem project "Nano" AntwerpHasselt, funded by the Flemish Government.

\section{References}

1 T. H. Borst and O. Weis, Diamond Relat. Mater., 1995, 4, 948-953.

2 W. Gajewski, P. Achatz, O. Williams, K. Haenen, E. Bustarret, M. Stutzmann and J. Garrido, Phys. Rev. B: Condens. Matter Mater. Phys., 2009, 79, 045206.

3 P. Achatz, W. Gajewski, E. Bustarret, C. Marcenat, R. Piquerel, C. Chapelier, T. Dubouchet, O. Williams, K. Haenen, J. Garrido and M. Stutzmann, Phys. Rev. B: Condens. Matter Mater. Phys., 2009, 79, 201203.

4 S. Janssens, P. Pobedinskas, J. Vacik, V. Petrakova, B. Ruttens, J. D'Haen, M. Nesladek, K. Haenen and P. Wagner, New J. Phys., 2011, 13, 083008.

5 E. A. Ekimov, V. A. Sidorov, E. D. Bauer, N. N. Mel'nik, N. J. Curro, J. D. Thompson and S. M. Stishov, Nature, 2004, 428, 542-545.

6 N. Dubrovinskaia, R. Wirth, J. Wosnitza, T. Papageorgiou, H. Braun, N. Miyajima and L. Dubrovinsky, Proc. Natl. Acad. Sci. U. S. A., 2008, 105, 11619-11622.

7 B. Willems, V. Dao, J. Vanacken, L. Chibotaru, V. Moshchalkov, V. I. Guillamon, H. Suderow, S. Vieira, S. Janssens, O. Williams, K. Haenen and P. Wagner, Phys. Rev. B: Condens. Matter Mater. Phys., 2009, 80, 224518.

8 This program can be downloaded from www.eelsmodel.ua.ac.be.

9 J. Verbeeck and S. Van Aert, Ultramicroscopy, 2004, 101, 207-224.

$10 \mathrm{P}$. Trebbia and N. Bonnet, Ultramicroscopy, 1990, 34, 165-178.

11 M. Nelhiebel, P. H. Louf, P. Schattschneider, P. Blaha, K. Schwarz and B. Jouffrey, Phys. Rev. B: Condens. Matter Mater. Phys., 1999, 59, 12807.

12 K. Schwarz, P. Blaha and G. K. H. Madsen, Comput. Phys. Commun., 2002, 147, 71-76.

13 T. Wojewoda, P. Achatz, L. Ortega, F. Omnes, C. Marcenat, E. Bourgeois, X. Blase, F. Jomard and E. Bustarret, Diamond Relat. Mater., 2008, 17, 1302-1306.

14 V. Brazhkin, V. E. Ekimov, A. Lyapin, S. Popova, V. A. Rakhmanina, V. S. Stishov, V. Lebedev, Y. Katayama and K. Kato, Phys. Rev. B: Condens. Matter Mater. Phys., 2006, 74, 140502.

15 H. Kim, S. Rodriguez, M. Grimsditch, T. R. Anthony and A. K. Ramdas, Phys. B, 1999, 273-274, 624-627.

16 H. J. Kim, A. K. Ramdas, S. Rodriguez, M. Grimsditch and T. R. Anthony, Phys. Rev. Lett., 1999, 83, 4140.

17 S. A. Kajihara, A. Antonelli, J. Bernholc and R. Car, Phys. Rev. Lett., 1991, 66, 2010.

18 J. Nakamura, E. Kabasawa, N. Yamada, Y. Einaga, D. Saito, H. Isshiki, S. Yugo and R. C. C. Perera, Phys. Rev. B: Condens. Matter Mater. Phys., 2004, 70, 245111.

19 H. Idrissi, S. Turner, M. Mitsuhara, B. Wang, S. Hata, M. Coulombier, J. P. Raskin, T. Pardoen, G. Van Tendeloo and D. Schryvers, Microsc. Microanal., 2011, 17, 983-990.

20 S. Turner, O. I. Lebedev, O. Shenderova, I. I. Vlasov, J. Verbeeck and G. Van Tendeloo, Adv. Funct. Mater., 2009, 19, 2116-2124.

21 T. L. Daulton, D. D. Eisenhour, T. J. Bernatowicz, R. S. Lewis and P. R. Buseck, Geochim. Cosmochim. Acta, 1996, 60, 4853-4872.

22 J. Birrell, J. A. Carlisle, O. Auciello, D. M. Gruen and J. M. Gibson, Appl. Phys. Lett., 2002, 81, 2235-2237.

23 S. Bhattacharyya, O. Auciello, J. Birrell, J. A. Carlisle, L. A. Curtiss, A. N. Goyette, D. M. Gruen, A. R. Krauss, J. Schlueter, A. Sumant and P. Zapol, Appl. Phys. Lett., 2001, 79, 1441-1443.

24 R. F. Egerton, Electron Energy-Loss Spectroscopy in the TEM, Plenum Press, New York and London, 1996.

25 R. Brydson, L. M. Brown and J. Bruley, J. Microsc., 1998, 189, 137144.

26 M. Dadsetani, J. Titantah and D. Lamoen, Diamond Relat. Mater., 2010, 19, 73-77.

27 P. May, W. Ludlow, M. Hannaway, P. Heard, J. Smith and K. Rosser, Diamond Relat. Mater., 2008, 17, 105-117.

28 A. Barnard and M. Sternberg, J. Phys. Chem. B, 2006, 110, $19307-$ 19314. 